Research Article

\title{
Construction of Corporate Investment Decision Support Model Based on Deep Learning
}

\author{
Jian-tao Song (iD) \\ School of Finance and Accounting, Yellow River Conservancy Technical Institute, Kaifeng 475004, China \\ Correspondence should be addressed to Jian-tao Song; songjiantao@yrcti.edu.cn
}

Received 15 July 2021; Revised 12 August 2021; Accepted 30 August 2021; Published 6 September 2021

Academic Editor: Bai Yuan Ding

Copyright (c) 2021 Jian-tao Song. This is an open access article distributed under the Creative Commons Attribution License, which permits unrestricted use, distribution, and reproduction in any medium, provided the original work is properly cited.

\begin{abstract}
Aiming at the problem of corporate investment decision support, this paper proposes and constructs a stock quality evaluation model based on deep learning and applies it to the stock quality evaluation of e-commerce enterprises. Firstly, LSTM neural network is used to construct the evaluation and prediction model. Secondly, the evaluation index system is constructed. Finally, the structure and parameters of the model are designed, and the prediction model is tested and evaluated through simulation experiments. The experiments prove that the model is reasonable and feasible, which can provide a reference for investors to make decisions.
\end{abstract}

\section{Introduction}

In recent years, with the development of the Internet, the e-commerce industry has developed rapidly, but the development of e-commerce is uncertain, and the industry has great competitiveness and bears great risks. The emergence of stocks has a certain impact on enterprise investment, and the stock market is also uncertain and risky. Therefore, it is of great significance for enterprises and investors to study stocks in the field of e-commerce. If a stock quality evaluation model is established to evaluate e-commerce enterprises reasonably, it is beneficial for the state to supervise the stock market and provide decision support reference for investors. With the development of computer technology, deep neural network is widely used in stock model research, and its structural characteristics are suitable for stock problem analysis. Yang et al. [1] proposed that deep learning should be used to improve the momentum strategy of stock market, and its improvement effect is good; Zeng and Nie [2] proposed a stock recommendation system based on deep learning, which is helpful for stock investment; Zhang et al. [3] proposed using deep learning algorithm to predict stock prices and provide effective reference for investors. On the basis of the above research, this paper puts forward a company investment decision support model based on deep learning and applies it to the evaluation of e-commerce stock quality, so as to correctly analyze the stock development of e-commerce enterprises and provide reference for investors. Literature [4] aimed at the rapid development of e-commerce today; whether the decision-making is accurate or not directly affects the operating effect of e-commerce companies. Investment decision-making system is an important way for investors to earn profits, and it is a model to support companies. By studying the data of e-commerce and Internet, combined with relevant theories, this paper puts forward a prediction model of corporate decision quality evaluation based on deep learning algorithm. It effectively provides support for investors in decision-making. According to the development direction and current state of domestic and foreign e-commerce investment market, literature [5] puts forward the modeling idea of investment decision quality evaluation index system and completes the investment decision support model by using modeling and simulation tools. Portfolio replication is realized by extracting the features of portfolio model by automatic encoder. Python programming language and library have been applied and implemented in the classification model of deep neural network (DNN) in literature [6]. Compared with traditional classification methods, deep learning classification model has better effect, because its accuracy can 
change with the size of data, thus increasing. There are many shortcomings in the traditional portfolio theory in literature [7], such as the construction of optimal socially responsible investment. Nowadays, people are more interested in how to invest in the environment, governance, and other aspects of the company, that is, socially responsible portfolio. Deep Responsibility Portfolio (DRIP) model uses reinforcement learning technology to balance the portfolio repeatedly, so as to achieve socially responsible portfolio. According to the data results, the latter is more competitive and has better social impact than the traditional portfolio model. Literature [8] aims at connecting suppliers and customers and uses the intelligent functions provided by cloud manufacturing platform and various processing schemes generated to effectively help customers choose manufacturing services. Its characteristic is that the more suppliers, the more schemes to choose from and the more complicated the customer's decision. Literature [9] aimed at the unstoppable trend of economic globalization; investors need to examine the reliability of solutions in the process of sound financial strategy, which leads to the intelligent decision support system. It uses mathematical tools of game theory and can consider many factors when making decisions on the same and similar problems. The rapid development of this kind of algorithms and components promotes the development of models based on the properties of bilinear differential games with multiple terminal planes. In literature [10], for real estate development companies must select high-performance and competitive cities as development projects, real estate asset evaluation is the top priority of regional strategy. The primary driving force for real estate investors is whether the region has urban competitiveness to attract all kinds of investment. The application of the comprehensive complex evaluation model to practical cases shows that it can map the multidimensional factor spectrum of the attractiveness of alternative real estate choices. According to literature [11], every investor expects investment to be proportional to income. Clear investment is a longer-term plan, with low or medium risks and low or medium returns. Investors' decisions will be influenced by market and psychology. The concept of investment decision support technology establishes a time frame for each goal, allows people to better choose the tools to achieve the goal, and defines the expected return or risk, investment portfolio, and the steps in the decision-making process of investment analysis. In view of the entertainment and commerce matched with the development of tourism economy, literature [12] analyzes the current situation of enterprises from the three-dimensional perspective of enterprise strategic portfolio and uses deep learning to analyze the impact of different portfolio characteristics on the operation and development of enterprises. It has been proved by practice that there are obvious differences between combination characteristics and single characteristics in enterprise decision-making and income. This paper puts forward the analysis of the impact of different combination policies on macroeconomy, and the forecast preparation rate of combination is as high as $98.57 \%$. In view of the complexity of investment decision analysis application fields and expert systems and the inability to provide accurate evaluation, literature [13] proposes a large-scale project investment decision system based on natural language evaluation and applies it to cloud model. Furthermore, a multiprocessing and multigranularity language decision-making system is proposed to support investment decision-making. The current situation of the research is explained, only the neural network is applied to various scenarios, and no industrialization decision is studied. The applications of these algorithms are based on a single neural network and have poor prediction results. Deep learning model is of important research significance for corporate investment, which can reduce investment risk and improve economic efficiency. This paper first introduces the LSTM algorithm, explains the application of deep network model in stock quality evaluation, and finally studies the stock forecast to analyze the investment decision behavior.

\section{LSTM Neural Network Algorithm}

BP neural network algorithm, RNN model, and LSTM algorithm can all be used in the training of evaluation and prediction model, in which BP neural network algorithm can only input the original data network one by one and cannot show the time series relationship between data during training and can only carry out error back-propagation layer by layer and cannot minimize the convergence of the network when dealing with gradient descent [14]. Although RNN model can input multiple original data at the same time and consider the time series relationship, it cannot solve the problem of gradient disappearance; LSTM algorithm is a special recurrent neural network. The information of its hidden layer can be transmitted to the output end or the hidden layer at the next time. LSTM algorithm can also update the weight continuously through input, output, and forgetting operations to avoid gradient expansion and disappearance. Compared with BP neural network algorithm, RNN model, and LSTM algorithm, the advantages of LSTM are that it has long-time memory and is simple to implement; the problems of gradient vanishing and gradient explosion existing during long sequence training are solved. This paper chooses LSTM algorithm to build the model, and its model structure is shown in Figures 1 and 2.

The training steps of LSTM algorithm are divided into four steps:

Step 1: Calculate the output of LSTM hidden layer. Let $w, o_{t}, c$, and $f$ denote output gate, cell state, forgetting gate, and input gate, respectively, let $b$ denote offset, let $w$ denote weight coefficient matrix, and $T(\tan h)$ and $S$ (sigmoid) are activation functions; then the expressions of forgetting gate and input layer are shown in the two following equations (1) and (2) [15]:

$$
\begin{aligned}
f_{t} & =\sigma\left(w_{f}\left[h_{t-1}, x_{t}\right]+b\right), \quad f_{t} \in[0,1] . \\
i_{t} & =\sigma\left(w_{i}\left[h_{t-1}, x_{t}\right]+b_{i}\right), \\
c 1_{t} & =\tan h\left(w_{c}\left[h_{t-1}, x_{t}\right]+b_{c}\right) .
\end{aligned}
$$

Update the cell state: 


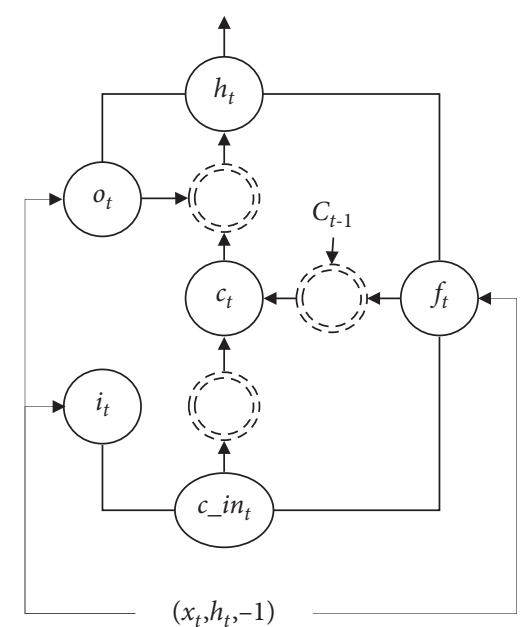

Figure 1: LSTM hidden layer structure diagram.

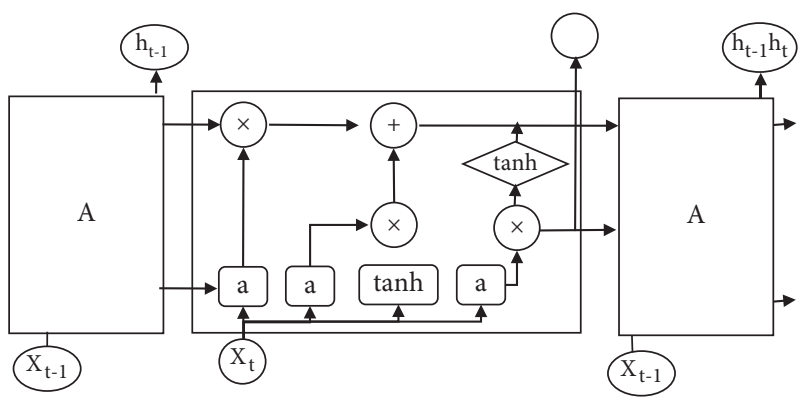

FIgURE 2: LSTM algorithm structure diagram.

$$
c_{t}=f_{t} * c_{t-1}+i_{t} * c 1_{t}
$$

The model output is obtained by activating the function:

$$
\begin{aligned}
& o_{t}=\sigma\left(w_{o}\left[h_{t-1}, x_{t}\right]+b\right), \\
& h_{t}=o_{t} * \tan h\left(c_{t}\right) .
\end{aligned}
$$

Step 2: The error $\delta$ of each LSTM cell is calculated by back-propagation, including the error terms in time and network level. Let $\delta t$ denote the error term at time $t$, let $\delta t-1$ denote the error term at time $t-1$, and let $\delta$ $k$ denote the error term at time $k$; then the delay reverse transmission of the error term is expressed as [16]

$$
\begin{aligned}
\delta_{t}^{T} & =\frac{\partial E}{\partial h_{t}}, \\
\delta_{t-1}^{T} & =\frac{\partial E}{\partial h_{t-1}} \\
\delta_{k}^{T} & =\prod_{j=k}^{t-1} \delta_{o, j}^{T} w_{o h}+\delta_{f, j}^{T} w_{f h}+\delta_{i, j}^{T} w_{c h} .
\end{aligned}
$$

Error terms are reversely transmitted to the upper layer along the network:

$$
\delta=\left(\delta_{f, t}^{T} w_{f x}+\delta_{i, t}^{T} w_{i x}+\delta_{o, t}^{T} w_{o x}\right) \cdot f^{\prime}\left(n e t_{t}^{l-1}\right)
$$

Step 3: Calculate the weight gradient according to the error [17].

$$
\begin{aligned}
\frac{\partial E}{\partial w_{o h}} & =\sum_{j=1}^{t} \delta_{o, j} h_{j-1}^{T}, \\
\frac{\partial E}{\partial w_{f h}} & =\sum_{j=1}^{t} \delta_{f, j} h_{j-1}^{T}, \\
\frac{\partial E}{\partial w_{i h}} & =\sum_{j=t}^{t} \delta_{i, j} h_{j-1}^{T}, \\
\frac{\partial E}{\partial w_{c h}} & =\sum_{j=1}^{t} \delta_{c, j} h_{j-1}^{T}, \\
\frac{\partial E}{\partial w_{o}} & =\sum_{j=1}^{t} \delta_{o, j}, \\
\frac{\partial E}{\partial w_{i}} & =\sum_{j=1}^{t} \delta_{i, j}, \\
\frac{\partial E}{\partial w_{f}} & =\sum_{j=1}^{t} \delta_{f, j},
\end{aligned}
$$

$w$ is the error with the fastest value at the point. In the decision of the company in the multi-index decision for error calculation, it finds out the index with the fastest change.

Step 4: Update the weights based on the previous step.

\section{Application of Deep Network Model in Stock Quality Evaluation}

3.1. Data Sources. The data of this paper comes from an information website. The stocks of 500 e-commerce companies are selected as experimental samples, and the sample stocks are divided into excellent stocks, common stocks, and loss stocks, with a ratio of $1: 1: 1$. The price-earnings ratio on the last day of 16 years is taken as the basis for differentiation. The sample stock annual reports from 2014 to 2016 were downloaded, and 30 indicators and 12,946 pieces of data were found in these annual reports. Excel 2017, Python data processing software, and TensorFlow system framework were adopted.

3.2. Indicator Selection. At present, there are many stock evaluation indexes in the market, such as net asset income and price-earnings ratio. Because stocks have risks and volatility, they can only be used as reference. In this paper, the price-earnings ratio is used as a reference to classify the sample data. In order to find the hidden connection of the company and grasp the development status of the enterprise, 
the existing indicators are not added to the evaluation system. When selecting indicators, we should follow compliance, scientificity, quantification, and feasibility so as to correctly analyze the stock quality of enterprises. The evaluation indicators selected in this paper include the following: asset-liability ratio, earnings per share, growth rate of operating income, proportion of R\&D investment, total share capital, number of circulating shares, issue price, number of senior executives, and 30 other indicators [18].

\subsection{Sample Data Processing}

3.3.1. Quantification of Nonnumerical Indicators. There are nonnumerical indexes in the index system, which must be transformed into numerical indexes if they are to be identified and processed by computer. In statistics, Likert scale is often used to transform text description into digital expression. There is no unified quantitative form in other disciplines, and the quantitative method is usually determined according to the characteristics and experience of indicators. Some indicators in this paper are expressed in the form of numbers and words, and there are three dimensions of expression. After consulting relevant data, this paper chooses the maximum-minimum standardization method to convert these indicators into numerical indicators. The specific operation is as follows: all nonnumerical indicators are standardized by maximum-minimum, and three dimensions are added to obtain one-dimensional index data.

3.3.2. Dimension Reduction Processing of Index Data. The correlation of all evaluation indexes is strong and weak. Dimension reduction is applied to the indexes; that is, the indexes with weak correlation are removed, which can improve the aggregation degree of the model and help to accurately evaluate the stock situation of enterprises. Let $r$ indicate the correlation of indexes; when $r<0.1$, it is not correlated, $0.1<r<0.3$ indicates weak correlation, $0.3<r<0.5$ indicates medium correlation, and $0.5<r<1$ indicates strong correlation. In this paper, PCA method is used to reduce the dimension of index data, and its steps are divided into three steps. First, after processing the sample data, it is converted into matrix form; the second is to calculate the index correlation coefficient matrix; the third is to show the index $r>0.1$, and calculate the variance percentage [19].

From this, we can get 18 of the 30 indexes with $R>0.3$, and then remove the indexes with $R<0.3$. By calculating the variance percentage of these 18 indexes, all of them can reach more than 95\%, which shows that PCA dimension reduction is feasible; see Figure 3.

3.3.3. Standardization of Indicator Data. Because the basic flow of the network model is to set the initial weight and input variables as the input of the hidden layer, get the output of the hidden layer through the activation function, and take it as the input of the next hidden layer, and operate repeatedly until the final network output. If the input data is

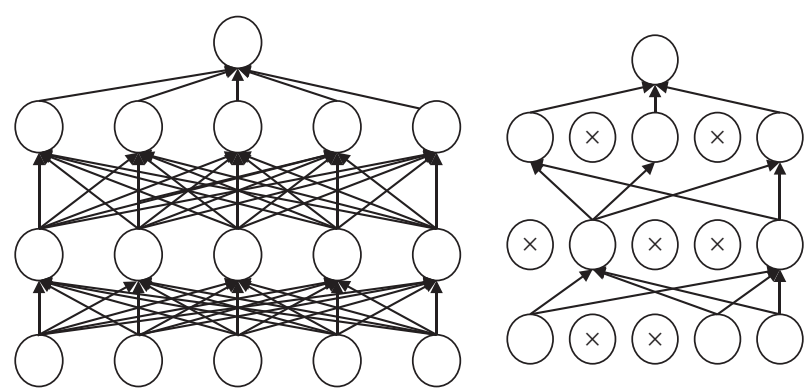

FIGURE 3: Schematic diagram of neural network after dimensionality reduction.

too large, under the action of activation function, the output data of network nodes may fall into the saturation interval, and the output of neurons will show the maximum or minimum value, resulting in the derivative gradually tending to 0 . In the process of error back-propagation, the modified parameter value is very small, the learning rate is slow, and the network cannot reach the convergence state. Therefore, this paper normalizes the data to prevent the paralysis of neural network caused by too large sample data. There are three commonly used methods for data normalization:

(1) Logarithmic function transformation method, the formula of which is [20]

$$
X_{i}^{\prime}=\log x_{i} .
$$

(2) Inverse cotangent function transformation method, the formula of which is

$$
X_{i}^{\prime}=\frac{2 \tan ^{-1} x_{i}}{\pi}
$$

(3) Linear function transformation method, the formula of which is

$$
X_{i}^{\prime}=\frac{x-x_{\min }}{x_{\max }-x_{\min }}
$$

According to the characteristics of samples, this paper adopts the linear function transformation method. Its principle is to calculate the maximum and minimum values of the original data and subtract the original data $x$ from the minimum value to correspond to $[0,1]$.

3.3.4. Discretization of Indicator Data. The purpose of discretization of index data is to evenly distribute the values of each piece of index data in the data samples, thus reducing the risk of overfitting and making the model have strong stability. Discretization is robust and can deal with abnormal data in sample data. Therefore, discretization method is usually used to deal with multiple continuous data in sample data. There are three main methods of discretization, namely, optimization and isometric and equal frequency discretization, among which optimization includes information gain and chi-square test, isometric means that the sample data is divided into $\mathrm{N}$ equal parts and the interval of 
each sample data is the same, and equal frequency means that the frequency of divided sample data is the same.

By observing and analyzing the original data, It is found that the original data is too large, and there are many continuous data and abnormal data, which will lead to the inability of the training model to fit the actual results. Therefore, it is necessary to discretize and standardize the sample data and train the processed data at the same time, so as to get a better model.

In this paper, the continuous data of 15 pieces of index data are discretized, and the stock quality types are divided into three grades by equidistance dispersion method, with values of 0,1 , and 2, respectively. After the data is standardized and discretized, the data is input into the network and then trained several times according to the training steps of LSTM algorithm, so that the number of hidden layer nodes is determined to be 50 , and the whole processing process is realized by Python language.

When evaluating the stock quality of enterprises, 18 indicators are selected as input variables: dividend, asset liability ratio, three-year earnings per share from 2014 to 2016, three-year reorganization, issue price, shareholding ratio of shareholders, listing time, number of legal proceedings, key pollutant discharge units, social responsibility report, total share capital, and number of circulating shares. Select 300 groups of training sample data, 200 groups of test sample data, and stock category as the evaluation output.

\subsection{Establishment of LSTM Neural Network Evaluation and Prediction Model}

3.4.1. Structural Design of Prediction Model. The input layer and output layer of neural network model are set according to data characteristics and targets, while other parameters are set according to experience or experiment, without fixed procedures and steps, which reflects the freedom of network model structure design. The hierarchy of neural network is generally set according to experience, and the research shows that the hierarchy is small, and its network popularization is strong, which is convenient for extracting features. The hierarchy is large, the network error is small, and the accuracy is high, but, at the same time, it also increases the complexity, the training speed, and time of the model, which may lead to overfitting phenomenon. Therefore, the number of hierarchies can be set according to the effect that the model needs to achieve. Generally speaking, in order to make the model have higher accuracy, we choose more than three layers of networks, and this paper chooses 10 layers of LSTM network to build the stock quality evaluation prediction model.

The sample data contains 500 stocks, and each stock has 18 pieces of data. According to the parameter setting basis of the input layer, the output layer, and the hidden layer, the input layer node number $m$ is set to 18 , the output layer point number $n$ is set to 3 , and the trial-and-error method is adopted to calculate the initial value of the hidden layer node number, assuming that $s$ represents the hidden layer node number and $a$ represents a constant, and the trial-and-error method expression is shown in formula (11), and the hidden layer node number is calculated to be 50 .

$$
\begin{aligned}
& s=\log _{2} m, \\
& s=\sqrt{m n} ; \\
& s=\sqrt{m+n}+a .
\end{aligned}
$$

3.4.2. Selection of Activation Function. Tanh, ReLU, and sigmoid are all active functions. When the neural network is small, Tanh has the best expressive force, while ReLU is the most widely used active function at present. Compared with sigmoid function and Tan $h$ function, the gradient of ReLU function has no saturation force, but it can quickly adjust parameters and alleviate the gradient disappearance process, and the calculation speed is faster. Therefore, ReLU function is selected as the activation function, and its expression is as follows (Figure 4):

$$
f(x)=\max (0, x) .
$$

\section{Simulation Experiment}

4.1. Original Data Processing Results. The original data are standardized and discretized, and the results are given in Tables 1 and 2 .

The stock quality type is divided into three grades with the values of 0,1 , and 2 . The value range is evenly divided into 3 equal parts, with equal spacing. It mainly helps LSTM to realize its process and can submit the prediction effect.

Take the above data as network input, and train it repeatedly according to LSTM training steps, and get the number of hidden layer nodes as 50 . The whole simulation experiment is completed by Python language.

4.2. The Results are Implemented in Python. Python is commonly used open-source software, which can combine languages $\mathrm{C}, \mathrm{C}++$, Java, and so forth and put packages not written by Python language into Python programming platform, which can identify and read packages. Python itself also has packages and extension libraries, including various functions inside, which can perform fast operations on numeric arrays.

4.2.1. Simulation Experiment Steps. There are seven steps in the simulation experiment. The first step is to define the file path of storage model, training set and test set; the second step is to define an LSTM single layer with a "switch"; the third step is to set up a multilayer LSTM network; the fourth step is to use dynamic_rnn function to make tf build a multilayer LSTM network and define the output; the fifth step is to define the loss function and train the neural network through the optimizer of tf.train; the sixth step is to solve the problem of overfitting; and the seventh step is to train LSTM neural network according to parameters. 


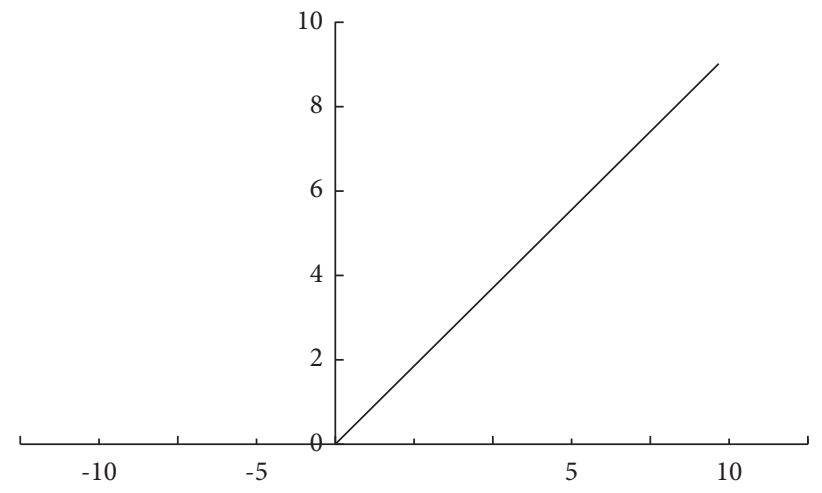

FIgURE 4: ReLU activation function.

TABle 1: Partial data after standardization.

\begin{tabular}{|c|c|c|c|c|c|c|c|c|c|}
\hline Code & Industry & Capital & Ating cap & Property & Oclcholder & To market & Price & Add stock & Profit \\
\hline 1 & 0.667 & 0.032 & 0.035 & 1 & 0.302 & 0.333 & 0.052 & 1 & 0.129 \\
\hline 2 & 0.33 & 0.001 & 0.001 & 1 & 0.329 & 0.250 & 0.055 & 0 & 0.005 \\
\hline 3 & 1.000 & 0.001 & 0.002 & 0 & 0.110 & 0.917 & 0.193 & 1 & 0.046 \\
\hline 4 & 0.667 & 0.010 & 0.012 & 0 & 0.410 & 0.750 & 0.177 & 1 & 0.355 \\
\hline 5 & 0.333 & 0.001 & 0.001 & 0 & 0.327 & 0.917 & 0.225 & 0 & 0.074 \\
\hline 6 & 0.333 & 0.088 & 0.088 & 1 & 0.521 & 0.417 & 0.029 & 1 & 0.027 \\
\hline 7 & 0.333 & 0.000 & 0.000 & 1 & 0.224 & 0.250 & 0.072 & 0 & 0.074 \\
\hline 8 & 0.333 & 0.010 & 0.012 & 0 & 0.390 & 0.833 & 0.128 & 1 & 0.355 \\
\hline 9 & 0.333 & 0.005 & 0.006 & 1 & 0.342 & 0.792 & 0.541 & 0 & 0.428 \\
\hline 10 & 0.333 & 0.023 & 0.024 & 1 & 0.182 & 0.250 & 0.014 & 0 & 0.775 \\
\hline 11 & 0.333 & 0.002 & 0.002 & 0 & 0.426 & 0.542 & 0.030 & 0 & 0.022 \\
\hline 12 & 0.667 & 0.005 & 0.007 & 1 & 0.350 & 0.583 & 0.018 & 1 & 0.077 \\
\hline 13 & 0.667 & 0.186 & 0.186 & 1 & 0.252 & 0.833 & 0.019 & 0 & 0.027 \\
\hline 14 & 0.667 & 0.002 & 0.003 & 1 & 0.503 & 0.250 & 0.036 & 1 & 0.059 \\
\hline 15 & 0.667 & 0.006 & 0.010 & 0 & 0.415 & 0.792 & 0.528 & 0 & 0.01 \\
\hline 16 & 0.667 & 0.002 & 0.003 & 1 & 0.382 & 0.167 & 0.064 & 1 & 0.062 \\
\hline 17 & 0.333 & 0.008 & 0.008 & 0 & 0.490 & 0.917 & 0.051 & 0 & 0.034 \\
\hline 18 & 0.333 & 0.000 & 0.001 & 0 & 0.384 & 1.000 & 0.191 & 1 & 0.607 \\
\hline
\end{tabular}

TABLe 2: Partial data after discretization.

\begin{tabular}{lcccccc}
\hline General_capital & Floating_capital & Stock_holder & Time_to_market & Price & 2014_profit & 2015_profit \\
\hline 0 & 0 & 0 & 0 & 0 & 0 & 0 \\
0 & 0 & 1 & 0 & 0 & 0 \\
0 & 0 & 0 & 2 & 0 & 0 \\
0 & 0 & 1 & 2 & 0 & 1 \\
0 & 0 & 1 & 2 & 0 & 0 \\
\hline
\end{tabular}

4.2.2. The Running Result of the Data Model after Standardization and Discretization. After 5 times of standardization, the prediction accuracy of the model is only $46 \%$, and when the training is increased to 15 times, the prediction accuracy can reach $70 \%$. Then the test set data is input into the model, and the accuracy rate is $77 \%$, which shows that after the data is standardized, the simulation accuracy is not high. The fitting degree of the model after discretization can reach $92 \%$, which is higher than that after standardization, which shows that the training model has high reliability after discretization of the data.
4.2.3. Actual Forecast Results. Selecting the stocks of 25 companies, the index data as the network input into the model, the results show that the prediction accuracy of the model is about $75 \%$, indicating that about 19 stocks have the accuracy of quality prediction. Compared with other related models, the stock evaluation prediction model in this paper improves the evaluation accuracy, and the model is feasible.

Through the above analysis, it is known that there are 18 indicators that have strong correlation with stock quality evaluation; these 18 pieces of index data are input into the stock quality evaluation model, and, after repeated training 
of the model, the stock quality evaluation and prediction results are obtained, and the results are accurate. According to the prediction results, the enterprise is comprehensively analyzed to evaluate the development status and investment potential of the enterprise, thus providing a reference for investors and deciding whether to give decision support.

\section{Conclusion}

This paper designs and constructs a company investment decision support model based on deep learning and applies it to the evaluation of enterprise stock quality. In this study, LSTM multilayer neural network is used to establish the model, and the parameters in the input layer, output layer, and hidden layer of the network are set reasonably. At the same time, the model is trained repeatedly through simulation experiments, and the trained model is used to evaluate the test sample data, and the evaluation effect is good, which verifies the feasibility of the model. Due to my limited knowledge and technology, this paper needs further indepth study. The existing problems in the present stage are as follows: First, when large-scale data analysis is applied to the company's investment decision support model, it takes a certain amount of time to verify its economic feasibility. Second, deep learning requires large-scale server operation, especially for real-time data acquisition and time effectiveness of analysis. The focus of future research work can solve the timeliness of data analysis, analyze all kinds of data in parallel, and then conduct joint analysis.

\section{Data Availability}

The experimental data used to support the findings of this study are available from the corresponding author upon request.

\section{Conflicts of Interest}

The author declares that there are no conflicts of interest regarding this work.

\section{References}

[1] Q. Yang, X. Wang, and Q. Tang, "Research on momentum strategy of Chinese a-share market based on deep learning method," Journal of Guangxi University of Finance and Economics, vol. 32, no. 3, pp. 36-55, 2019.

[2] A. Zeng and W. Nie, "Stock recommendation system based on deep bidirectional LSTM," Computer Science, vol. 46, no. 10, pp. 84-89, 2019.

[3] Q. Zhang, D. Yan, and J. Han, "Research on stock price forecasting based on deep learning and decomposition algorithm," Computer Engineering and Application, vol. 57, no. 5, pp. 56-64, 2021.

[4] Z. Lei, "Research and analysis of deep learning algorithms for investment decision support model in electronic commerce," Electronic Commerce Research, vol. 20, no. 2, pp. 275-295, 2020.

[5] L. Hou, "Decision support model of e-commerce enterprise investment based on deep learning algorithm," Information Systems and E-Business Management, 2021.
[6] S. Bayraci and O. Susuz, "A deep neural network (DNN) based classification model in application to loan default prediction," Theoretical and Applied Economics, vol. 621, no. 4, pp. 75-84, 2019.

[7] N. N. Y. Vo, X. He, S. Liu, and G. Xu, "Deep learning for decision making and the optimization of socially responsible investments and portfolio," Decision Support Systems, vol. 124, Article ID 113097, 2019.

[8] A. Simeone, A. Caggiano, B. Deng, and L. Boun, "A deep learning based-decision support tool for solution recommendation in cloud manufacturing platforms," Procedia CIRP, vol. 86, pp. 68-73, 2019.

[9] V. Lakhno, V. Malyukov, B. Akhmetov, N. Gerasymchuk, H. Mohylnyi, and P. Kravchuk, "Decision support model for assessing projects by a group of investors with regards of multi-factors," in Artificial Intelligence and Bioinspired Computational Methods. CSOC 2020, R. Silhavy, Ed., vol. 1225, pp. 1-10, Springer, Cham, 2020.

[10] V. D. Giudice, P. D. Paola, T. Francesca, P. J. Nijkamp, and A. Shapira, "Real estate investment choices and decision support systems," Sustainability, vol. 11, no. 11, pp. 1-18, 2019.

[11] S. Gupta and L. N. Das, "Optimal investment decision model based on simplex algorithm with variable optimal value evaluation process," ICACM 2018. Springer Proceedings in Mathematics \& Statistics, vol. 320, pp. 433-443, Springer, Singapore, 2020.

[12] B. Li, "Construction of business strategic planning structure model based on deep learning algorithm," Information Systems and E-Business Management, vol. 25, pp. 1-18, 2021.

[13] X. Mi, H. Liao, and X. J. Zeng, "Investment decision analysis of international megaprojects based on cognitive linguistic cloud models," International Journal of Strategic Property Management, vol. 24, no. 6, pp. 414-427, 2020.

[14] y. Fu, "Intelligent financial decision making: integration of soft computing and machine learning," Financial Research, no. 6, pp. 31-36, 2019.

[15] Q. Yang, G. Zhang, and B. Ma, "Management semantics and the risk of future stock price crash: an analysis of mediating effect based on investor sentiment," Journal of Zhongnan University of Economics and Law, no. 1, pp. 26-36, 2020.

[16] Q. Han, G. Cai, and Z. Zhao, "Research on the growth evaluation method of small and medium-sized enterprises based on big data technology," Statistics and Information Forum, vol. 34, no. 7, pp. 123-128, 2019.

[17] X. Tang, M. Tan, and S. Li, "Research on the model construction and implementation of enterprise bankruptcy prediction system," Journal of Information Science, vol. 38, no. 10, pp. 1051-1065, 2019.

[18] H. Zhang, H. Shen, and Y. Liu, "Research on multi factor quantitative stock selection based on self attention neural network," Mathematical Statistics and Management, vol. 39, no. 3, pp. 556-570, 2020.

[19] X. Niu and W. Zonghao, "Research on multi factor strategy of Chinese stock market based on Integrated Learning," Journal of Guizhou Party School, vol. 20, no. 4, pp. 22-37, 2020.

[20] T. Lin, Q. Zhang, and X. Qi, "Securities big data analysis and research," Computer Technology and Development, vol. 30, no. 10, pp. 179-186, 2020. 